\title{
Viabilité de l'élevage pastoral au Sahel : étude de quelques paramètres démographiques des élevages de Hombori (Mali)
}

\author{
Mamadou O. Diawara ${ }^{1,2, *}$, Pierre Hiernaux ${ }^{2}$, Eric Mougin $^{2}$, Fabrice Gangneron $^{2}$ et Nogmana Soumaguel ${ }^{3}$ \\ ${ }^{1}$ Département de biologie, faculté des sciences et techniques (FST), université des sciences, des techniques et des technologies de \\ Bamako (USTTB), Colline de Badalabougou, BP 3206 Bamako, Mali \\ ${ }^{2}$ Géosciences environnement Toulouse (GET), observatoire Midi-Pyrénées, université de Toulouse, CNRS, IRD, 14, avenue Edouard \\ Belin, 31400 Toulouse cedex, France \\ ${ }^{3}$ Centre IRD de Bamako, Quartier Hippodrome, BP 2528 Bamako, Mali
}

\begin{abstract}
Résumé - L'élevage pastoral représente la principale activité économique de nombreux ménages au nord du Sahel. Malgré la qualité des pâturages au cours de la saison des pluies, cet élevage est soumis à de nombreuses contraintes liées à l'irrégularité de la disponibilité fourragère, aux difficultés d'accès aux compléments alimentaires et aux soins vétérinaires qui rendent précaires les productions animales. Or la demande en produits d'origine animale est en forte augmentation du fait de la croissance démographique soutenue et de la rapide urbanisation au Sahel et dans les pays côtiers. Cette étude à pour objectif de documenter les paramètres démographiques des troupeaux et la production fourragère des parcours utilisés par l'élevage pastoral de la commune de Hombori située au nord-est du Sahel malien. La démarche adoptée est basée sur des enquêtes rétrospectives sur la composition spécifique des troupeaux et les évènements démographiques qui l'affectent. Ces enquêtes ont été conduites du 15 juillet au 15 septembre 2011 auprès de 120 ménages résidants dans la commune. Les résultats mettent en évidence une faible productivité des troupeaux de conduite pastorale en rapport avec le niveau des paramètres de reproduction, notamment l'âge tardif à la première mise bas, qui s'explique par des pratiques pastorales très extensives. Ils montrent également que les taux d'exploitation sont élevés, en particulier pour les ovins, alors même que les performances reproductrices sont faibles et les taux de mortalité élevés. Pour améliorer la productivité des troupeaux, assurer la pérennité de ces systèmes d'élevage et la transmission intergénérationnelle du cheptel, la qualité et surtout la régularité saisonnière de l'alimentation du bétail doivent être améliorées à travers une plus grande mobilité pastorale mais aussi une supplémentation saisonnière ciblée.
\end{abstract}

Mots-clés : productivité animale / pratiques pastorales / production fourragère des parcours / viabilité économique / Sahel

\begin{abstract}
Visibility of pastoralism in the Sahel: study of demographic parameters of livestock herds in Hombori (Mali). Pastoralism is the main economic activity for many households in northern Sahel. Despite rangeland quality during the rainy season, pastoral husbandry is subject to many constraints related to the irregular availability of forage and the lack of access to food supplements and veterinary care making livestock production precarious. Yet the market demand for livestock products is growing rapidly triggered by high population growth and rapid urbanization in the Sahel states and also in the neighboring coastal states. This study aims to document the herd demographic parameters and the forage production of rangelands used by livestock from the district of Hombori located in the northeast of Malian Sahel. The approach is based on retrospective surveys on herd composition and demographic events conducted from July 15 to September 152011 with 120 households from the district. The results indicate a low productivity of pastoral husbandry related to poor reproductive parameters including the late age at first calving, linked to very extensive pastoral practices. They also show strong exploitation rates particularly for sheep flocks even when reproductive performance are low and mortality rates are high. To improve herd productivity, ensure the sustainability of these pastoral systems and the intergenerational transmission of livestock capital, the quality of livestock nutrition should be improved especially in seasonal regularity through greater pastoral mobility but also targeted seasonal supplementation.
\end{abstract}

Keywords: animal productivity / pastoral practices / forage production / economic viability / Sahel

\footnotetext{
*Auteur correspondant : diaprod@hotmail.com
} 


\section{Introduction}

L'élevage constitue l'une des principales activités économiques du Sahel avec une contribution de 30 à $40 \%$ aux Produits intérieurs bruts agricoles de pays comme le Burkina Faso, le Cap-Vert, le Mali, la Mauritanie, le Niger, le Sénégal, le Soudan et le Tchad (Mulumba et al., 2008). En plus de cette participation à l'économie, l'élevage pastoral constitue l'un des principaux modes de production agricole au Sahel. Les pays sahéliens disposent d'un important potentiel de production de viande avec un cheptel estimé en 2006 à 63 millions de bovins, 168 millions de petits ruminants et plus de 6 millions de camelins (Dicko et al., 2006).

Basé sur la pâture des parcours, et de quelques jachères, l'élevage dans cette région repose sur des disponibilités fourragères fortement dépendantes des aléas climatiques et des variations saisonnières de la pression de pâture comme l'ont montré les grandes sécheresses des années 1970 et 1980 (Toulmin, 1985). Ces sécheresses ont causé la perte de près de $80 \%$ du cheptel de la région et entraîné le déplacement de milliers de personnes (Toulmin, 1985).

Malgré l'ampleur des pertes en bétail, la succession d'années humides à partir de 1994, bien que ponctuée d'années localement sèches comme celles de 2004 et 2008 (Lebel et Ali, 2009), a permis la reconstitution numérique du cheptel (Pradère, 2007). Au Mali, les effectifs de bovins ont ainsi augmenté de $30 \%$ entre 1990 et 2005 , et ceux des petits ruminants de $26 \%$ (Mulumba et al., 2008).

Cette hausse des effectifs de bétail, doublée d'une mobilité pastorale restreinte, oblige les animaux à mobiliser davantage leurs réserves adipeuses (Pullan, 1980). En plus des pertes saisonnières de poids qui caractérisent ce type d'élevage et des risques de mortalité, ces contraintes peuvent également affecter la capacité reproductrice des femelles, en particulier l'âge de la première mise bas et l'allongement des intervalles entre deux parturitions (Blanc et al., 2006).

Alors que la hausse nationale des effectifs de bétail au cours des vingt dernières années est confirmée par plusieurs auteurs, il semble que la productivité des troupeaux s'affaiblit (Pradère, 2007). Cependant, les paramètres de reproduction sont peu documentés et la viabilité de l'élevage pastoral naisseur au Sahel demeure mal évaluée. Les seules statistiques disponibles sur les effectifs de bétail sont issues des campagnes de vaccination et elles sont à la fois éparses et très peu fiables (Wint et Bourn, 1994). À cela s'ajoute la rareté des données démographiques sur les élevages pastoraux. L'élevage pastoral à Hombori comme partout ailleurs au Sahel devrait profiter de l'opportunité liée à l'accroissement de la demande en produits animaux à l'échelle régionale tout en s'adaptant à un milieu soumis aux aléas évoqués précédemment.

La présente étude a pour objectif d'évaluer la productivité mais aussi la viabilité de l'élevage pastoral au Sahel à travers le cas d'élevages de familles résidentes de la commune de Hombori située au nord-est du Mali.

\section{Matériels et méthodes}

\subsection{Sites d'étude}

Les données utilisées ont été collectées auprès de 120 ménages de Hombori, l'un des quatre sites étudiés dans le cadre du projet ECliS (Élevage Climat et Société). Les ménages se répartissent entre 9 villages qui ont été choisis de façon à capter la diversité des systèmes d'élevage pastoraux résidants (Fig. 1). Ces villages forment deux grands ensembles en fonction de l'activité principale du chef de ménage.

Le premier ensemble est constitué de villages agropastoraux (Béria, Garmi, Dossou, Bilantao et Kiri). Dans ces villages, malgré les faibles rendements, l'activité dominante est la culture du mil. L'élevage y est essentiellement ovin et caprin avec des troupeaux de petite taille. Outre ces deux activités, le commerce de bétail et l'artisanat constituent les autres sources de revenus pour ces populations essentiellement sédentaires.

L'autre ensemble que forment les villages pastoraux de Darawal, Agoufou, Kelma et Tondibongho, se caractérise par l'élevage comme activité dominante. Les parcours de la commune de Hombori sont aussi exploités de juillet à février par des troupeaux transhumants en provenance des plaines du Macina, saisonnièrement inondées par la crue annuelle du fleuve Niger (Wilson, 1988), mais aussi des régions mitoyennes à l'ouest (Haïré, Seno Mango), au sud (Djelgobé) et au nord (Gourma). Ces éleveurs et leurs troupeaux ne sont pas inclus dans l'échantillon étudié à cause de l'irrégularité et de la saisonnalité de leur présence sur la commune. Les enquêtes réalisées dans l'échantillon montrent l'existence de tensions autour de l'accueil de ces troupeaux qui seraient responsables d'exploitation «abusive» des ressources pastorales.

\subsection{Les méthodes d'enquête}

La collecte des données s'est appuyée sur des méthodes d'enquêtes rétrospectives : la méthode 12MO (12 mois) et la méthode des carrières de reproductrices (Lesnoff, 2011). La méthode 12MO développée par le Centre de coopération internationale en recherche agronomique pour le développement (CIRAD) et l'International Livestock Research Institute (ILRI), est une méthode d'enquêtes rétrospectives pour l'estimation systématique de paramètres démographiques: composition du troupeau par sexe et classes d'âge, taux de fécondité, de mortalité et les flux d'entrées et sorties sur l'année. Basée sur des entretiens avec les éleveurs, elle permet de reconstituer la démographie du troupeau durant les 12 derniers mois ayant précédé l'enquête sur la base des animaux entrés et sortis du troupeau. Les enquêtes sont réalisées en une visite au cours de laquelle le troupeau doit être sur place.

L'enquête sur la carrière de femelles permet d'estimer des paramètres de reproduction des femelles, de préférence nées dans le troupeau sur une période rétrospective qui s'étend à toute la vie de la reproductrice jusqu'à la date de l'enquête. Les taux démographiques dits naturels sont estimés d'après les mises bas déclarées et les taux de mortalité et d'exploitation à partir du devenir des jeunes issus de ces mises bas (Lesnoff, 2011). Ces enquêtes ont été conduites dans la commune de Hombori entre le 15 juillet et le 15 septembre 2011. Les paramètres de reproduction ont été établis sur un sous échantillon de 134 vaches, 35 brebis et 49 chèvres. Ils portent sur l'âge de la femelle, le nombre de mises bas au cours de la carrière et au cours des 12 derniers mois précédant l'enquête, le nombre d'avortements au cours de la carrière et au cours de la 


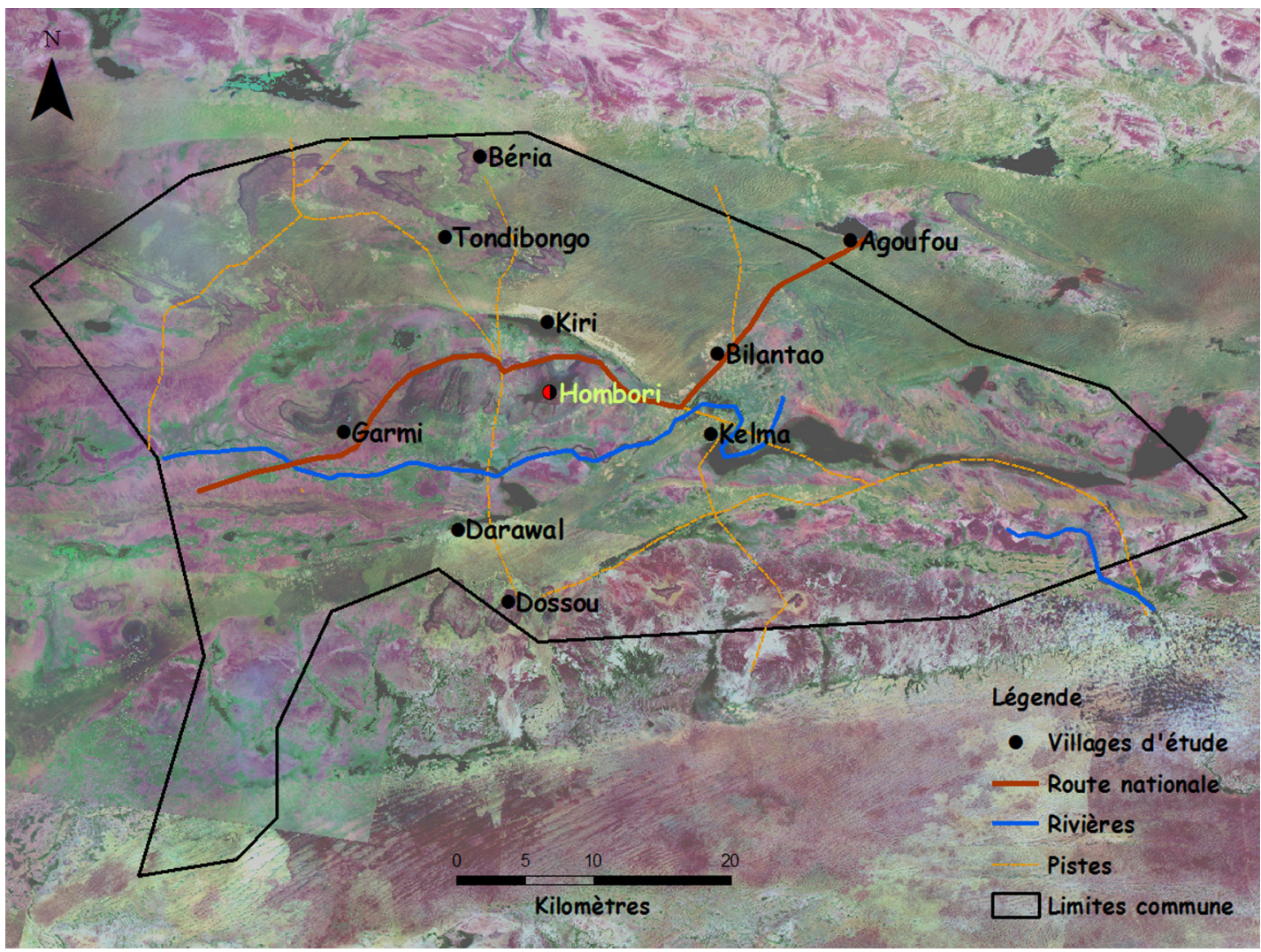

Fig. 1. Localisation des villages de l'étude dans la commune de Hombori.

Fig. 1. Localisation of study villages in the Hombori district.

même période, le nombre de nés vivants mâles ou femelles, le nombre de mort-nés et nombre de jeunes morts, le devenir de chaque jeune. Les autres flux d'entrées et de sorties d'animaux ont été également caractérisés par des enquêtes (12MO) pour la même période. Le décompte des sorties d'animaux a été fait par espèce et par catégorie d'âge en distinguant mortalité, abattage, perte, don de l'éleveur (zakât) et vente. Les gains étant ceux dus aux naissances dans le troupeau, aux dons à l'éleveur ou aux achats (Lesnoff, 2011).

\subsection{Analyse des données}

Les paramètres démographiques des troupeaux ont été estimés à partir des données collectées. Le calcul de la productivité des troupeaux $(R)$ repose sur l'équation proposée par Lesnoff (2011) qui s'appuie sur les paramètres démographiques recueillis et calculés précédemment:

$$
R=\frac{\left(\Delta_{n}+O-I\right)}{N_{m}}
$$

Dans cette équation, $\Delta_{n}$ représente la variation de stock au cours de l'année (potentiel exploitable moins la production effective), $O$ et $I$, les animaux respectivement exploités (abattages, ventes, dons cédés) et importés (achats, dons reçus) dans le troupeau au cours de l'année, $N_{m}$ étant l'effectif moyen du troupeau au cours de l'année. Le potentiel exploitable représente les naissances, dont on déduit les mortalités et les pertes au cours de l'année. La production effective étant l'exploitation (abattages, ventes et dons cédés) moins les importations (achats et dons reçus). La variation du stock rapportée à l'effectif moyen estime le taux de croît annuel du troupeau $(T c)$.

$$
T c=\frac{\Delta_{n}}{N_{m}}
$$

La production nette du troupeau $(O-I)$ rapportée à l'effectif moyen estime le taux d'exploitation du troupeau (Tx) au cours de l'année (Lesnoff, 2011).

$$
T x=\frac{O-I}{N_{m}} .
$$

Pour les pratiques pastorales, un certain nombre de variables ont été utilisées pour élaborer une typologie des pratiques pastorales pour laquelle une analyse factorielle des correspondances multiples, puis une classification ascendante hiérarchique, ont été réalisées. Les variables retenues pour cette analyse sont: les espèces élevées, le statut du troupeau (gestion directe ou confiage), son orientation (séparation en plusieurs catégories de gestion ou conduit en un seul bloc), la mobilité saisonnière, le recours à une complémentation, les taux d'exploitation. Les autres variables (accès aux services vétérinaires, mode de surveillance du troupeau, difficultés 
Tableau 1. Typologie des pratiques pastorales à Hombori en 2011.

Table 1. Typology of pastoral practice to Hombori in 2011.

\begin{tabular}{|c|c|}
\hline Types d'exploitations & Modes de conduite des troupeaux \\
\hline \multirow[t]{6}{*}{ Type 1 (bovin-ovin-caprin) } & $63 \%$ des élevages de l'échantillon \\
\hline & Troupeaux sédentaires \\
\hline & Recours aux compléments alimentaires (tourteau de coton mais aussi les gousses \\
\hline & d'acacia pour les petits ruminants) \\
\hline & Importants flux d'entrées et de sorties d'animaux \\
\hline & $17 \%$ des bovins sont en confiage \\
\hline \multirow[t]{6}{*}{ Type 2 (élevages mono-spécifiques de caprins) } & $3,3 \%$ des élevages étudiés \\
\hline & Troupeaux de taille supérieure à la moyenne \\
\hline & Tous les animaux sont en propriété \\
\hline & Troupeaux sont sédentaires \\
\hline & Pas de recours aux compléments alimentaires \\
\hline & Entrées se font essentiellement par les naissances \\
\hline \multirow[t]{4}{*}{ Type 3 (élevages de petits ruminants) } & $5,8 \%$ des élevages de l'échantillon étudié \\
\hline & Troupeaux sont sédentaires \\
\hline & Pas de recours aux compléments alimentaires ni au fourrage aérien \\
\hline & Important flux d'entrées et de sorties \\
\hline \multirow[t]{5}{*}{ Type 4 (bovin-caprin) } & $10 \%$ des élevages étudiés \\
\hline & Seuls les caprins sont en propriété \\
\hline & Très peu de sorties d'animaux et elles portent essentiellement sur les caprins \\
\hline & Recours aux compléments alimentaires pour les bovins (tourteau de coton) \\
\hline & Troupeaux sédentaires \\
\hline \multirow[t]{6}{*}{ Type 5 (élevages mono-spécifiques de bovins) } & $6,7 \%$ des élevages \\
\hline & Troupeaux de grande taille ( 80 têtes en moyenne) \\
\hline & Séparation en plusieurs catégories de gestion en milieu de saison sèche \\
\hline & Compléments alimentaires pour la fraction qui reste au point d'attache \\
\hline & Mobilité pour l'autre fraction \\
\hline & Entrées d'animaux se font essentiellement par les naissances \\
\hline
\end{tabular}

d'accès aux parcours) ont été écartées à cause de leur faible participation à la distinction des types de pratiques pastorales.

\section{Résultats}

\subsection{Les troupeaux}

Les enquêtes ont permis d'échantillonner 109 troupeaux de bovins, 96 troupeaux d'ovins et 105 troupeaux de caprins. Les élevages sont mixtes le plus souvent et la configuration la plus répandue est l'association des trois espèces qui représente $68 \%$ des élevages de l'échantillon étudié. À l'opposé, on note l'absence d'élevage monospécifique d'ovins. Les effectifs moyens et leurs écart-types des troupeaux étudiés sont de $48 \pm 41$ têtes pour les bovins, $28 \pm 20$ têtes pour les ovins et $39 \pm 29$ têtes pour les caprins. Dans ces troupeaux, tous les animaux ne sont pas en propriété et les taux de confiage représentent $19 \%$ des effectifs de bovins, $7 \%$ des effectifs d'ovins et $6 \%$ des effectifs de caprins.

\subsection{Modes de conduite des troupeaux}

La composition spécifique des troupeaux nécessitant des modes de conduite propres à chaque exploitation, il a été possible d'identifier cinq types de pratiques pastorales (Tab 1). L'élevage de la commune repose sur l'exploitation des parcours et très secondairement des résidus de cultures. La mobilité du bétail de la commune s'exerce dans un rayon limité $(15$ à $20 \mathrm{~km})$, ce qui justifie un recours fréquent à la complémentation alimentaire. La durée de cette complémentation, les quantités distribuées et les catégories d'animaux qui reçoivent ces rations varient énormément en fonction des exploitations. La stratégie de normalisation des observations que nous avons utilisée a été de ramener les quantités distribuées au nombre de femelles adultes dans l'exploitation. En 2011, le niveau de complémentation observé dans les exploitations de l'échantillon étudié était de $0,7 \pm 0,5 \mathrm{~kg} / \mathrm{jour}$ de tourteau de coton, par femelle adulte pendant $120 \pm 54$ jours.

\subsection{Paramètres démographiques}

\subsection{1 Âge à la première mise bas (APMB)}

L'âge moyen au premier vêlage est de $60 \pm 13$ mois $(n=134)$ avec un coefficient de variation de $22 \%$. Cet âge varie en fonction de la race, qui explique $20 \%$ des variations totales. Il est de $61 \pm 12$ mois pour les zébus Maures, de $47 \pm 10$ mois pour les zébus Peuhls et de $71 \pm 13$ mois pour les zébus Bororos. Globalement, l'âge d'entrée en reproduction est indépendant du troupeau d'appartenance de la vache. L'âge moyen au premier vêlage est supérieur à ceux observés par Tamboura et al. (1982) et Wagenaar et al. (1986) dans des 
élevages du centre du Mali. L'âge au premier agnelage est de $16 \pm 6$ mois $(n=35)$ avec un coefficient de variation de $39 \%$. Pour les caprins, l'âge à la première mise bas est de $14 \pm 4$ mois $(n=49)$ avec un coefficient de variation de $30 \%$. Ces âges sont très proches de ceux observés par Wilson (1988) dans des systèmes pastoraux proches de celui de Hombori. Contrairement aux bovins, l'âge à la première mise bas varie beaucoup entre les troupeaux pour les troupeaux de petits ruminants, ce qui explique $28 \%$ à $30 \%$ des variations observées respectivement chez les ovins et les caprins.

\subsubsection{Intervalle entre parturitions}

L'intervalle entre deux parturitions est de : $25 \pm 5,4$ mois pour les zébus Bororos, 21,7 7,7 mois pour les zébus Maures et de 18,8 $\pm 4,1$ mois pour les zébus Peuhls. Pour les petits ruminants, cet intervalle est de $11,9 \pm 2,6$ mois pour les ovins et $10,7 \pm 2,6$ pour les caprins. Ces amplitudes entre parturitions sont légèrement supérieures à celles observées par Wilson (1988) au centre du Mali avec $8,7 \pm 2,5$ et 9,7 $\pm 3,5$ mois, respectivement pour les ovins et les caprins.

\subsubsection{Taux d'avortement}

Les taux d'avortement moyens et les coefficients de variation suivants ont été enregistrés dans les troupeaux de l'échantillon : $2,43 \pm 11,2 \% ; 3,2 \pm 9,5 \%$ et $18,7 \pm 99 \%$ respectivement pour les bovins, ovins et caprins. Le taux d'avortement des caprins est élevé et le coefficient de variation observé de $99 \%$ est dû à la perte de la quasi-totalité des portées d'un des troupeaux à la suite de l'introduction d'un nouveau géniteur. Ce genre de pratique qui vise «l'amélioration génétique » est courante et n'est pas sans danger. En effet, les mâles achetés sur le marché peuvent être porteurs de germes de maladies abortives comme la brucellose ou la fièvre Q qui peuvent anéantir la productivité annuelle, voire de plusieurs années. Les taux d'avortement calculés dans l'étude sont conformes à ceux rapportés dans d'autres travaux réalisés au Sahel. Wilson (1988) a observé au centre du Mali des taux d'avortement de $12,6 \%$ pour les caprins et $5,1 \%$ pour les ovins. Dans la même région, Wagenaar et al. (1986) ont enregistré entre 1979 et 1983 un taux moyen d'avortement de $6,7 \%$ pour les bovins.

\subsubsection{Taux de prolificité}

Les taux de prolificité des trois espèces sont de 1,00 pour les bovins, 1,04 et 1,14 respectivement pour les ovins et les caprins. Les chèvres de notre échantillon ont une prolificité comparable à celle d'autres chèvres sahéliennes telles que celles observées par Wilson (1988) au centre du Mali qui ont une prolificité de 1,19. Elle est aussi inférieure à celle observée par Robinet (1967) pour des chèvres rousses de Maradi $(1,44)$. Les prolificités des vaches et des brebis de notre échantillon s'apparentent aussi à celles observées ailleurs au Sahel dans des conditions d'élevage proches de celles de Hombori (Haumesser, 1975 ; Wilson et Light, 1986; Niaré, 1996).

\subsubsection{Taux de mise bas}

Pour les bovins de l'échantillon, le taux moyen annuel de mise bas est de $57 \%$. Ce taux se situe dans la fourchette des taux observés au Sahel. Il est inférieur à ceux rapportés par Wilson et Wagenaar (1983) au Niger, respectivement 69 et $63 \%$, mais supérieur aux $50 \%$ observés par Wagenaar et al. (1986) au Mali. Chez les ovins, le taux moyen de mise bas de $83 \%$ observé n'est pas exceptionnel au Sahel, mais ce taux est bas, comparé à ceux rapportés par Haumesser et Gerbaldi (1980) et Dumas (1980) dans des études réalisées respectivement au Niger et au Tchad. Les chèvres de l'échantillon ont un taux de mise bas moyen de $93 \%$. Ce taux est supérieur à la plupart des taux enregistrés ailleurs au Sahel (Tourrand et Landais, 1996). Cependant, Haumesser dans une étude réalisée au Niger en 1975 a enregistré un taux largement supérieur à celui de notre étude $(114 \%)$.

\subsubsection{Taux de mortalité}

En moyenne, les taux de mortalité juvénile $(<1$ an) observés dans les exploitations de notre échantillon, s'élèvent à $8 \%$ pour les bovins, $9 \%$ pour les ovins et $15 \%$ pour les caprins. Ces taux bruts de mortalité des petits ruminants sont inférieurs à ceux observés par Wilson (1988) à partir d'un suivi réalisé au centre du Mali entre 1978 et 1984 . La cause la plus fréquemment évoquée par les éleveurs est une maladie foudroyante (la cowdriose, «Mberdè» en langue peuhl), qui frappe toutes les espèces. Sur la base des déclarations des éleveurs, cette maladie expliquerait $65 \%$ des mortalités des bovins. Sur la base des mêmes déclarations, les stress nutritionnels sont responsables de $25 \%$ des mortalités d'ovins, $10 \%$ des mortalités de caprins et $3 \%$ des mortalités de bovins.

\subsection{Productivité des troupeaux et dynamique des élevages}

La productivité annuelle des trois principales espèces est présentée en fonction des types de pratiques pastorales (Fig. 2). Élevés seuls ou en association avec d'autres espèces, les caprins de l'échantillon ont une productivité numérique supérieure à celle des deux autres espèces. Les caprins élevés en association avec des ovins (troisième type) dégagent une productivité numérique de $46,1 \%$. Les ovins de l'échantillon sont peu productifs et ont des taux de croît négatifs, ce qui pourrait justifier leur association systématique aux deux autres espèces. La productivité numérique des bovins de l'échantillon est très variable. Conduits seuls (cinquième type) ou en association avec des ovins et des caprins (premier type), les bovins dégagent une productivité numérique supérieure à $12 \%$ alors que ceux du quatrième type conduit avec les caprins ont une productivité numérique presque nulle malgré le recours aux compléments alimentaires.

Les taux annuels d'exploitation des petits ruminants sont élevés dans l'échantillon, respectivement $21,9 \%$ et $20,2 \%$ pour les ovins et les caprins (Tab 2). Le taux annuel d'exploitation des bovins s'élève à $7,8 \%$. Les ventes constituent le premier facteur de sortie des animaux dans les troupeaux. Elles représentent $83,6 \%$ des sorties de bovins, $74,4 \%$ et $78,1 \%$ respectivement pour les ovins et les caprins. Les autres sorties sont dues aux abattages. Malgré cette exploitation modérée, le croît annuel moyen des troupeaux en 2011 est faible pour les caprins $(5,7 \%)$ et négatif pour les bovins et les ovins, respectivement à $-3,5 \%$ et $-5 \%$. 

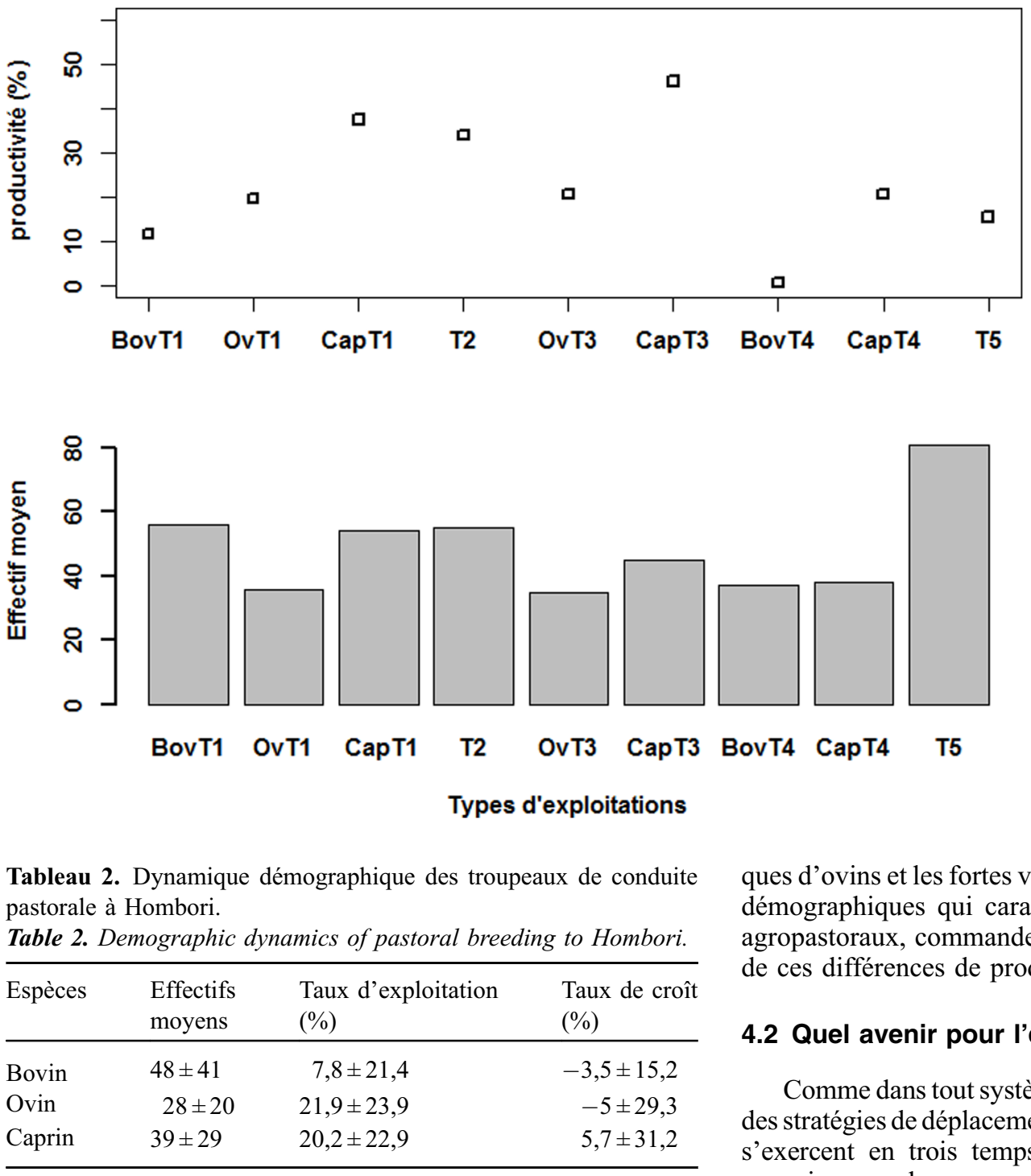

\section{Discussion}

\subsection{Démographie des troupeaux}

La différence de productivité entre les exploitations révèle l'importance des pratiques pastorales sur la productivité des troupeaux. Les bovins du quatrième type sont en confiage, l'éleveur n'est pas le gestionnaire alors que dans les deux autres types, il a tout ou partie du cheptel bovin en propriété et il est donc maître de toutes les décisions de gestion. La perte d'effectif de bovins et d'ovins observée dans l'échantillon est due aux fortes mortalités enregistrées en 2010 malgré une production fourragère estimée à $1845 \pm 748 \mathrm{~kg} \mathrm{MS} / \mathrm{ha}$ sur le territoire de la commune de Hombori et ses environs.

D'autres facteurs comme la race ou la performance exceptionnelle de certaines femelles, liée à leur potentiel génétique, peuvent également expliquer cette différence de productivité entre exploitations (Clément et al., 1997). Cependant, le manque de recul dans le temps sur les flux d'animaux, la taille restreinte de l'échantillon, les effectifs restreints des troupeaux, l'absence d'élevages monospécifi-
Fig. 2. Productivité annuelle par espèce en fonction de la taille des troupeaux et des types de pratiques pastorales. Bov, Ov et Cap désignent respectivement, les bovins, les ovins et les caprins. T suivi d'un numéro désigne le type de pratiques pastorales d'appartenance.

Fig. 2. Annual productivity (\%) of each species depending on the herds size and pastoral practice types. Bov, $O v$, Cap mean respectively cattle, sheep and goats. $T$ followed by number means the type of pastoral practices of belonging. ques d'ovins et les fortes variations temporelles des paramètres démographiques qui caractérisent les systèmes pastoraux et agropastoraux, commandent la prudence dans l'interprétation de ces différences de productivité entre exploitations.

\subsection{Quel avenir pour l'élevage pastoral au Sahel?}

Comme dans tout système pastoral, les pasteurs ont toujours des stratégies de déplacement. Celles que nous avons observées s'exercent en trois temps, elles sont basées sur une bonne connaissance des espaces et/ou des formes d'appropriation territoriale qui méritent attention. Notons par exemple qu'à la saison des pluies les animaux sont tous éloignés de la couronne villageoise cultivée, pour exploiter les espaces pastoraux ouverts. Lorsque les récoltes sont faites, les animaux pâturent résidus de culture et adventices. À l'épuisement de ces ressources, on peut indiquer deux types de stratégies.

La première concerne les groupes à dominante pastorale, ils conduisent les troupeaux au maximum à un jour ou deux de marche, dans le Séno au sud de la commune.

La seconde stratégie concerne les groupes à dominante agropastorale de l'échantillon. Dans quelques-uns de ces cas nous avons pu observer que les villageois pratiquent une « mise en réserve » d'espaces péri-villageois entre la zone de culture et celle pâturée en saison sèche (Gangneron, 2013). Ils restent donc inexploités par les animaux du village et par les pasteurs transhumants jusqu'en saison sèche chaude. Ces cas sont peu répandus car ils supposent une maitrise villageoise de ces espaces qui leur sont généralement contestés par l'ensemble des pasteurs et plus particulièrement par les grands transhumants.

Une autre stratégie activée en saison sèche, le recours à la supplémentation, est également bridée par les prix pratiqués 
sur les compléments alimentaires peu ou pas subventionnés qui les rendent peu accessibles.

\section{Conclusion}

En dépit du caractère ponctuel de l'enquête et du mode de recueil des données basées sur la mémoire des éleveurs, les paramètres démographiques estimés dans cette étude sont en accord avec ceux observés dans des systèmes pastoraux sahéliens similaires à ceux de Hombori.

L'étude montre que l'élevage pastoral se maintient malgré les faibles rendements des troupeaux dus aux modestes performances de reproduction: première mise bas tardive, longs intervalles entre mise bas et faibles taux de prolificité, associés à des taux de mortalité et d'avortement variables entre les troupeaux et dans le temps mais globalement élevés. Les mortalités et pertes par avortement ont souvent une cause infectieuse mais cette morbidité est aggravée par l'état de sousnutrition saisonnière caractéristique du Sahel. Dans ces conditions, une plus grande mobilité saisonnière des troupeaux est une voie pour optimiser l'utilisation des ressources pastorales et améliorer in fine la productivité des troupeaux.

Le recours à la supplémentation saisonnière et ciblée est une voie complémentaire pour maintenir la productivité de l'élevage. L'achat d'aliments du bétail se fait individuellement au détail et en période de crise en fin de saison sèche, alors que les prix sont au plus haut. Une politique d'achat coopératif en début de saison sèche devrait permettre de réduire le coût et de promouvoir le recours à la supplémentation. Cela permettrait de réduire l'impact des crises que peuvent engendrer des accidents climatiques tels que les sécheresses subies par les élevages de Hombori en 1972-1973 et en 1983-1984 et leur impact catastrophique sur le cheptel (Toulmin, 1985).

En dépit des crises traversées et de sa faible productivité, l'élevage pastoral perdure comme activité économique majeure des familles à Hombori. Il est vrai que ces familles ont adopté de longue date, en plus des stratégies pastorales, des stratégies de diversification d'activité. Suivant les groupes de population ces activités portent sur des cultures vivrières, mais aussi quelques cultures maraîchères, sur le commerce de bétail ou colportage, sur le bucheronnage et charbonnage, l'artisanat, le transport ou encore des membres de la famille ont recours à la longue tradition de migration saisonnière ou pluriannuelle de la région. C'est donc à la lumière de l'ensemble des activités pratiquées, à l'échelle de l'unité familiale que l'on parvient à comprendre la durabilité du système pastoral.

Remerciements. Les auteurs remercient le projet ANR-ECliS qui a financé en partie cette étude. Les auteurs remercient aussi les éleveurs de la commune de Hombori, ainsi que Boureima Cissé et Aly Maiga, pour leur coopération et leur accompagnement dans la conduite des enquêtes. Enfin, les auteurs remercient les relecteurs pour leurs nombreuses remarques.

\section{Références}

Blanc F, Bocquier F, Agabriel J, D'Hour P, Chilliard Y. 2006. Adaptative abilities of the females and sustainability of ruminant livestock systems. A review. Anim. Res 55: 489-510.
Clément V, Poivey JP, Faugère O, Tillard E, Lancelot R, Gueye A et al. 1997. Étude de la variabilité des caractères de reproduction chez les petits ruminants en milieu d'élevage traditionnel au Sénégal. Revue d'Élevage et de Médecine vétérinaire des Pays Tropicaux 50: 235-249. http://remvt.cirad.fr/cd/EMVT97_3.PDF

Dicko MS, Djitèye MA, Sangaré M. 2006. Les systèmes de production animale au Sahel. Sécheresse 17: 83-97.

Dumas R. 1980. Contribution à l'étude des petits ruminants du Tchad. Revue d'Élevage et de Médecine vétérinaire des Pays Tropicaux 33 (2): 215-233. http://remvt.cirad.fr/cd/EMVT80_2.PDF

Gangneron F. 2013. Ressources pastorales et territorialité chez les agro-éleveurs sahéliens du Gourma des buttes. VertigO 13 (3). DOI : $10.4000 /$ vertigo. 14427 .

Haumesser JB. 1975. Quelques aspects de la reproduction chez la chèvre rousse de Maradi : comparaison avec d'autres races tropicales ou sub-tropicales. Revue d'Élevage et de Médecine Vétérinaire des Pays Tropicaux 28 (2): 225-234. http://dx.doi.org/ 10.19182/remvt.8050

Haumesser JB, Gerbaldi P. 1980. Observations sur la reproduction du mouton Oudah nigérien. Revue d'Élevage et de Médecine vétérinaire des Pays Tropicaux 33 (2): 205-213. http://remvt. cirad.fr/cd/EMVT80_2.PDF

Lebel T, Ali A. 2009. Recent trends in the Central and Western Sahel rainfall regime (1990-2007). Journal of Hydrology 375 (1-2): 52-64.

Lesnoff M. 2011. Démographie et zootechnie tropicales: un lien par les modèles matriciels appliqués aux cheptels de ruminants dans les élevages extensifs. Mém. Rech. HDR, Université de Montpellier II, 233 p. https://agritrop.cirad.fr/563912/1/document_ 563912.pdf

Mulumba JBK, Somda J, Sanon Y, Kagoné H. 2008. Élevage et marché régional au Sahel et en Afrique de l'Ouest. Potentialités et défis. CSAO-OCDE/ CEDEAO, 182 p. https://www.oecd.org/fr/ csao/publications/40279092.pdf

Niaré T. 1996. Performances de reproduction des ovins dans deux noyaux d'élevage traditionnel et cycle fourrager en zone soudanosahélienne au Mali. Agronomie Africaine VIII (1): 41-50.

Pradère J. 2007. Performances et contraintes de l'élevage au Mali. Projet d'appui à l'agriculture africaine : amélioration des politiques agricoles dans les pays d'Afrique de l'ouest et du centre, FIDA, la France, OCDE et Hub Rural de Dakar, 73 p.

Pullan NB. 1980. Productivity of white Fulani cattle on the Jos plateau, Nigeria II. Nutritional factor. Trop. Anim. Health, Prod 12: $17-24$.

Robinet AH. 1967. La chèvre Rousse de Maradi. Son exploitation et sa place dans l'économie de l'élevage de la République du Niger. Revue d'Élevage et de Médecine Vétérinaire des Pays Tropicaux 20 (1): 129-186. http://remvt.cirad.fr/cd/EMVT67 1.PDF

Tamboura T, Bibe B, Babile R, Petit JP. 1982. Résultats expérimentaux sur le croisement entre races locales et races laitières améliorées au Mali. Revue d'Élevage et de Médecine Vétérinaire des Pays Tropicaux 35: 401-416. http://remvt.cirad.fr/cd/EMVT82_4.PDF

Toulmin C. 1985. Livestock losses and post-drought rehabilitation in sub-Saharan Africa. LPU working paper $\mathrm{N}^{\circ}$. International Livestock Centre for Africa, Addis Ababa, Ethiopia.

Tourrand JF, Landais E. 1996. Productivité des caprins dans les systèmes de production agricole du Delta du fleuve Sénégal. Revue d'Élevage et de Médecine vétérinaire des Pays Tropicaux 49 (2): 168-173. http://remvt.cirad.fr/cd/EMVT96_2.PDF

Wagenaar KT, Diallo A, Sayers AR. 1986. Productivity of transhumant Fulani cattle in the inner Niger delta of Mali ILCA Research Report no. 13. Adis Ababa, Ethiopia: ILCA (International Livestock Centre for Africa. 
Wilson RT. 1988. La production animale au Mali central : études à long terme sur les bovins et les petits ruminants dans le système agropastoral. ILRI.

Wilson RT, Wagenaar KT. 1983. An introductory survey of livestock population demography and reproductive performance in the area of the Niger Range and Livestock Project. Arid Zone Program Document No. AZ 80. Bamako, Mali: International Livestock Centre for Africa.
Wilson RT, Light D. 1986. Livestock production in central Mali: economic characters and productivity indices for traditionally managed goats and sheep. Journal of Animal Science 62 (3), $567-575$.

Wint W, Bourn D. 1994. Anthropogenic and environmental correlates of livestock distribution in sub-saharian Africa. A comparative analysis of livestock surveys in Mali, Niger, Nigeria, Sudan and Tchad. O.D. Administration, London, UK.

Citation de l'article : Diawara MO, Hiernaux P, Mougin E, Gangneron F, Soumaguel N. 2017. Viabilité de l'élevage pastoral au Sahel: étude de quelques paramètres démographiques des élevages de Hombori (Mali). Cah. Agric. 26: 45006. 